\title{
INTRA-URBAN MOVEMENT FLOW ESTIMATION USING LOCATION BASED SOCIAL NETWORKING DATA
}

\author{
A. Kheiri ${ }^{\text {a }}$, F. Karimipour ${ }^{\text {b,* }}$, M. Forghani ${ }^{\text {b }}$ \\ ${ }^{a}$ Faculty Technical and Engineering, Eslamic Azad University of Larestan, Iran - asma.kheiri@ymail.com \\ ${ }^{b}$ Faculty of Surveying and Geospatial Engineering, College of Engineering, University of Tehran, Tehran, Iran - \\ (fkarimipr, mo.forghani)@ut.ac.ir
}

KEY WORDS: Location Based Social Network, Check-in Data, O/D Matrix, Mobility

\begin{abstract}
:
In recent years, there has been a rapid growth of location-based social networking services, such as Foursquare and Facebook, which have attracted an increasing number of users and greatly enriched their urban experience. Location-based social network data, as a new travel demand data source, seems to be an alternative or complement to survey data in the study of mobility behavior and activity analysis because of its relatively high access and low cost. In this paper, three OD estimation models have been utilized in order to investigate their relative performance when using Location-Based Social Networking (LBSN) data. For this, the Foursquare LBSN data was used to analyze the intra-urban movement behavioral patterns for the study area, Manhattan, the most densely populated of the five boroughs of New York city. The outputs of models are evaluated using real observations based on different criterions including distance distribution, destination travel constraints. The results demonstrate the promising potential of using LBSN data for urban travel demand analysis and monitoring.
\end{abstract}

\section{INTRODUCTION}

An important part of human activities in an urban environment is their mobility behavior. Nowadays, measuring the movement of people is a fundamental activity in modern societies. First insight regarding the mobility within a region can be captured by extracting the origin-destination $(\mathrm{O} / \mathrm{D})$ matrix, which specifies the travel demands between the origin and destination nodes on a network. This matrix could be on different scales including macroscopic scales, e.g., at the inter-urban level, or at microscopic scales, e.g., at the intra-urban level. In the intraurban level, OD matrix is indicative of the movement of people between different areas of the city. Many methods have been suggested for OD-matrix estimation, which can be classified into three main categories (Jin et al., 2013): survey-based methods, traffic counts, and methods based on the positioning technology. Survey-based methods as traditional OD estimation methods such as telephone, in-person interview, mail or email survey are always time-consuming and costly tasks and they have limited sample sizes and lower frequencies. Moreover, the survey data cannot provide up-to-date information to reflect the rapid changes in travel demand pattern. Traffic count based methods calibrate an OD matrix based on traffic detector data (Jin et al., 2014). These methods are based on an existing metering infrastructure, which may be expensive to install or maintain. Moreover, estimation of OD matrices from this data is extremely challenging because the data is very often limited in extent, which can lead to multiple plausible non-unique OD matrices (Jin et al., 2013; Igbal et al., 2014). Using LBSN data for OD matrix estimation is a method based on the positioning technology which is raised in recent literatures as a new travel demand data source and has attracted an increasing number of users and significantly raised their urban experience. Location based social networking sites such as Foursquare allow a user to "check in" at a real-world POI (point of interest, e.g., a hotel, coffee shop, art gallery, etc.), leave tips about the POI, and share the check-in with their online friends.

Compared to traditional GPS data, location based social networks data have unique features with many information to reveal human mobility, i.e., "when and where a user (who) has been to for what," and the temporal check-in sequence of a specific person can be considered as his/her trajectory. Therefor, these information provides opportunities to better understand human mobility from spatial, temporal, social, and content aspects. Additionally, with the rapid growth of smartphones, the LBSN application can be easily built in personal mobiles and tablets without concerning the maintenance and update issues in the traditional traffic monitor infrastructure. The sample size can be much larger than other methods because the penetration rate of social networking service is growing at a rapid pace. Moreover this data has the potential to provide origin-destination movement estimation with significantly higher spatial and temporal resolution at a much lower cost in comparison with traditional methods.

On the other hand, despite the long history of modeling human mobility, predicting mobility patterns in cities has been a challenging task until now, and the lack of an accurate approach with low data requirements for predicting mobility patterns in cities can still be felt. So far, many of researchers have tried to predict intra-urban movement using mobile positioning technologies but the results show that there is not significant achievements (Calabrese et al., 2011).

This study examines the efficiency of LBSN check-in data provided by Foursquare in the estimation of the intra-urban OD matrix. The remainder of this paper is organized as follows: Section 2 introduces the used dataset and an initial analysis conducted for the characteristics of check-ins collected. The datafiltering steps are presented in Section 3. Section 4 presents OD estimation models and our proposed approach, which are evaluated using a MTA OD matrix in Section 5. Finally, Section 6 presents some discussions and conclusions.

\section{DATASET AND PRELIMINARY ANALYSIS}

\subsection{Review of the Dataset}

In this paper, Manhattan, the most densely populated of the five boroughs of New York city, is selected as the study area. 
Manhattan had a population of $1.6363 \mathrm{~m}$ people in July 2014 according to the U.S. Census Bureau estimate and encompasses an area of $87.46 \mathrm{~km}^{2}$. The data used in this study for analysis on the intra-urban movement can be categorized into three parts: the 2010 census tracts data of New York city from U.S. Census Bureau, New York Customer Travel Survey data from the Metropolitan Transportation Authority (MTA) ${ }^{1}$ and the checkins data from Foursquare.

The census tract data is used as spatial resolution to estimate movement distribution. There are 288 identified tracts within the borough of Manhattan, which will serve as the study area for this paper. MTA origin-destination survey data serves as the ground truth data used for comparison. The Survey provided a rich source of information about the tract to tract travel behavior of New York city residents from May through November 2008. This survey captured detailed information on the travel of 4,014 residents in 3,433 households in the borough of Manhattan. In this paper, Foursquare data set was chosen to study the human movement behavioral patterns based on geo-social networks because Foursquare is the most popular LBSN, with $31 \%$ of mobile users active on social networks using it. The check-ins data related to study area is extracted from source data. The resulted dataset contains 100,879 check-ins of 1083 users for about 10 month (from 12 April 2012 to 16 February 2013).

\subsection{Preliminary Analysis of the Check-ins Data}

In this sub-section, a preliminary analysis is conducted on the characteristics of the check-ins occurrence by investigating of the spatial pattern of the check-ins data. The spatial distribution of the 100,879 check-ins are represented using scatter dots in Figure 1(a). As shown in Figure 1(b), a heat map also represents the geographic density of check-ins features on study area by using graduated color areas to represent the quantities of those points. Also, according to the primary analysis, we found that the average number of check-ins per user is around 99 and the median is approximately 83 .

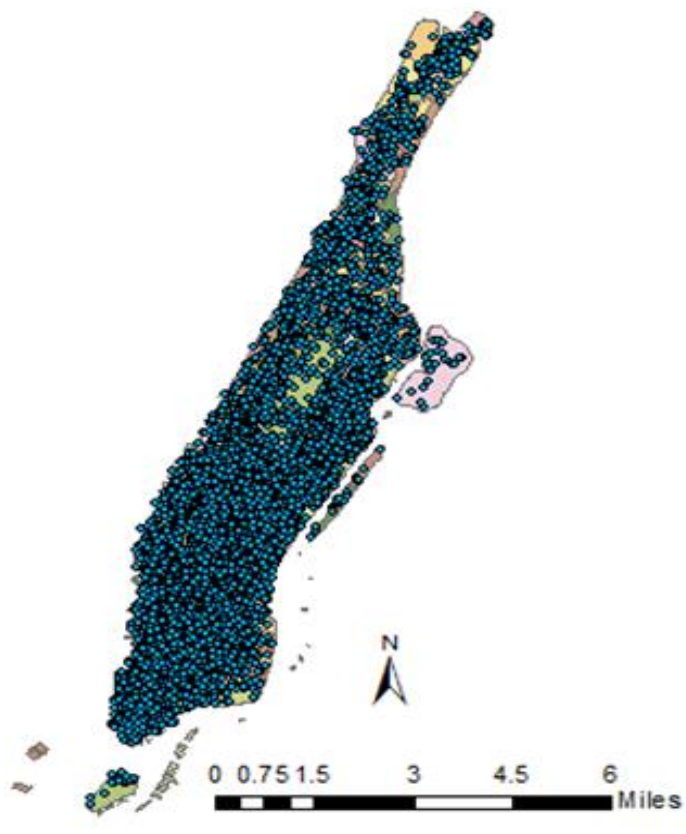

(a)

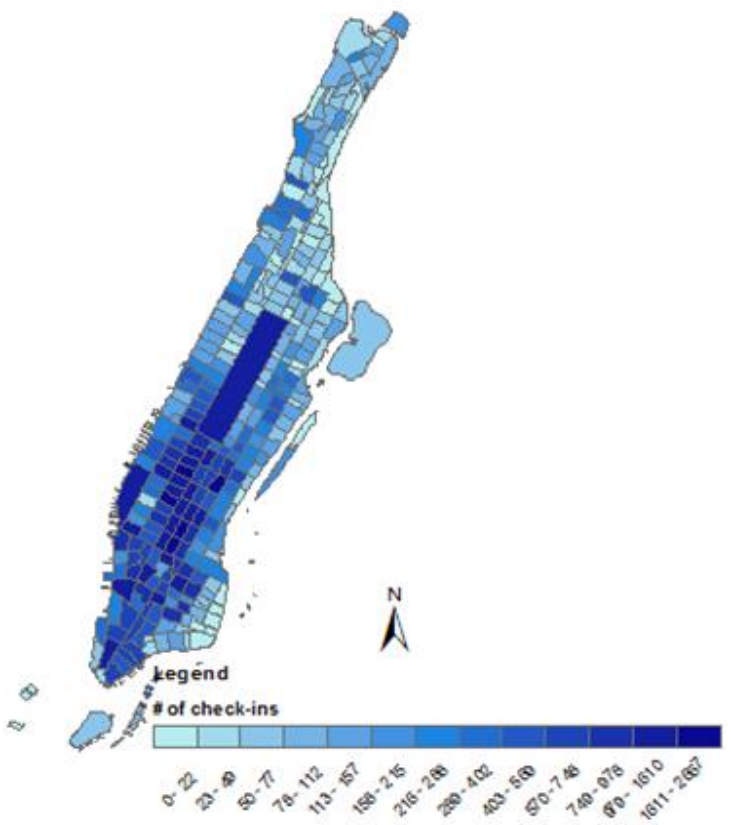

(b)

Figure 1. Foursquare check-ins locations and their spatial distribution among tracts

\section{FILTERING CHECK-IN RECORDS}

Although most social media services provide some mechanism to prevent the emergence of fake check-ins, invalid check-ins and trips still exist. Invalid check-ins prevent the efficiency of data for exploring intra-urban human mobility patterns and must be eliminated. This section presents how the check-in records and trips were filtered out and extracts daily trajectories from checkin data.

1) Filtering the duplicate check-ins: Some users check-in several times at the same location upon or after arrival, which must be filtered.
2) Filtering the user with only one check-in: The user with only one check-in were filtered out as they do not allow any movement analysis.

Moreover, having individual spatio-temporal trajectories extracted by connecting the consecutive check-ins, we filtered out trips according to the following rules:

3) Removing the trips with time intervals less than 1 minute and more than 12 hours: Very short trips ( $<1$ minute) are not so sueful ans so filtered out. In addition, if time intervals between previous check-in is more than $12 \mathrm{~h}$, this means that some trips are very likely to be missing, and may deviate the results, so they also be removed.

\footnotetext{
${ }^{1}$ http://web.mta.info/mta/planning/data-nyc-travel.html
} 
4) Removing trips with speed greater than $200 \mathrm{~km} / \mathrm{h}$. If the speed of the trip is more than $200 \mathrm{~km} / \mathrm{h}$, it means the user is traveling at a speed extremely higher than any urban transportation modes, including bus, subway, car, etc. Such trips were considered fake trips and were removed.

After applying the above filtering process, 69675 check-ins and 31978 trips were finally obtained.

\section{METHODOLOGY}

Since the 1940s, various models such as gravity model (Zipf, 1946), intervening opportunity model (Stouffer, 1940), radiation model (Simini et al., 2012), rank-based model (Noulas et al., 2012) and population-weighted opportunities (PWO) model (Yan et al., 2014) have been proposed for estimation of OD matrix. In this study, the three following models have been utilized to compare their performance for LBSN data analysis:

\subsection{Radiation Model}

The "radiation model" (Simini et al., 2012) defines a commuting flux from location $i$ to $j$ as:

$$
T_{i j}=T_{i} \frac{m_{i} n_{j}}{\left(m_{i}+s_{i j}\right)\left(m_{i}+n_{j}+s_{i j}\right)}
$$

where $m_{i}$ and $n_{j}$ are the population of locations $i$ and $j, T_{i}$ is the number of trips starting from $i$, and $s_{i j}$ is the total population in the circle of radius $r_{i j}$ (the distance between the origin $i$ and destination $j$ ) centered at location $i$ (excluding the source and destination population). This model needs only the spatial distribution of population as an input, without any adjustable parameters. Therefore, as an advantage, this model can be applied in areas where the previous mobility measurements are not available.

\subsection{Rank-based Model}

The "rank-based model" is inspired by the theory of intervening opportunities (Stouffer, 1940), which presents the rank distance as a key component, accounting in the number of places between origin and destination, rather than the pure physical distance. This model assumes that the probability of an individual travelling from an origin to a destination depends (inversely) only upon the rank-distance between the destination and the origin. The model is described as:

$$
T_{i j}=T_{i} \frac{R_{i}(j)^{-\gamma}}{\sum_{k \neq i}^{N} R_{i}(j)^{-\gamma}}
$$

where $R_{i}(j)$ is the rank-distance from location $j$ to $i$ (e.g., if $j$ is the closest location to $i, R_{i}(j)=1$; if $j$ is the second closest location to $i, R i(j)=2$, and so on) and is an adjustable parameter. This model belongs to the category of parameterized models. However, $\gamma$ presents minor variations from city to city. This model needs very low input information to reproduce some key characteristics of human mobility patterns.

\subsection{Population-weighted Opportunities Model}

Finally, the "population-weighted opportunities model" (Yan et al., 2014) assumes that the attraction of a destination is inversely proportional to the population $S_{j i}$ in the circle centered at the destination with radius $r_{i j}$ (the distance between the origin $i$ and destination $j$ ) minus a finite-size correction $1 / M$ :

$$
A_{j}=o_{j}\left(\frac{1}{s_{j i}}-\frac{1}{M}\right)
$$

where $A_{j}$ is the relative attraction of destination $j$ to travelers at origin $i, o_{j}$ is the total opportunities of destination $j$ and $M$ is the total population in the city. Hence, probability of travel from $i$ to $j$ is proportional to the attraction of $j$. Moreover, with the assumption that the number of opportunities $o_{j}$ is proportional to the population $m_{j}$, the travel from $i$ to $j$ is defined as:

$$
T_{i j}=T_{i} \frac{m_{j}\left(\frac{1}{s_{j i}}-\frac{1}{M}\right)}{\sum_{k \neq i}^{N} m_{k}\left(\frac{1}{S_{k i}}-\frac{1}{M}\right)}
$$

where $T_{i}$ is the trips starting from $i$ and $N$ is the number of locations in the city.

\subsection{Proposed Approach}

In order to evaluate the efficiency of LBSN data in the estimation of the intra-urban OD matrix using three mentioned models, firstly, Foursquare check-ins data for Manhattan, was collected and the collected raw data was filtered according to the approach described in section 3. Secondly, as each LBSN data record has a time attribute, check-ins were sorted based on time and individuals' trajectories were extracted using consecutive checkins. The study area was then partitioned based on census tracts and using aggregated trajectories between these areas, the movement flow intensity between each pair of tracts was estimated by each of the mentioned models. Although the mentioned models have no adjustable parameters, they require information on variables such as population distribution and location attraction as inputs. In order to extract these inputs from location-based social networking data and make the proposed models compatible with this kind of data, it is essential to renew and develop the models, considering specific characteristics and limitations of LBSN data. Therefore, in radiation and populationweighted opportunities, the number of check-ins were used instead of the population in the models. And in rank-based model, the number of venues that are closer in terms of distance to origin than destination were used instead of the rank-distance from location $j$ to $i$. In this paper, $\gamma$ was assigned the value 0.84 as proposed by (Noulas et al., 2012).

\section{EVALUATION OF MODELS}

This section compares the estimated Foursquare OD matrix using mentioned models with the MTA ground truth matrix. To evaluate the performance of the models we investigated the travel distance distribution by three models based on ground truth data. Travel distance distribution is an important statistical property to capture human mobility behaviours (Yan et al., 2014).

As shown in Figure (2) it can be found that the distribution of travel distance estimated by the rank-based model has a good accordance with the ground truth data compared to the other models. Moreover, we computed the probability of travel towards a location with population $m, P_{\text {dest }}(m)$, for both ground truth data and the models. As shown in Figure (3), the results of rankbased model have a better agreement with the ground truth data than those of the other models. Furthermore, in order to evaluate the similarity between the data obtained from the mentioned models and the ground truth data, we used Cosine and Sørensen similarity indices. Therefore, the O/D matrices were converted to vector form and cosine similarity method was used as follow: 


$$
\operatorname{Similarity}(i, j)=\cos (\vec{\imath}, \vec{\jmath})=\frac{\vec{\imath} \cdot \vec{\jmath}}{\|\vec{\imath}\| \times\|\vec{\jmath}\|}
$$

The Sørensen similarity index is a statistic tool to identify the similarity between two samples (Yan et al., 2014). In this paper, we were used the index to measure the degree of agreement between reproduced travel matrices and empirical observations. The index is defined as:

$$
S S I \equiv \frac{1}{N^{2}} \sum_{i}^{N} \sum_{j}^{N} \frac{2 \min \left(T^{\prime}{ }_{i j}, T_{i j}\right)}{{T^{\prime}}{ }_{i j}+T_{i j}}
$$

where $T^{\prime}{ }_{i j}$ is the travels from location $i$ to $j$ predicted by the model and $T_{i j}$ is the observed number of trips. The results of evaluation are presented in Table 1.

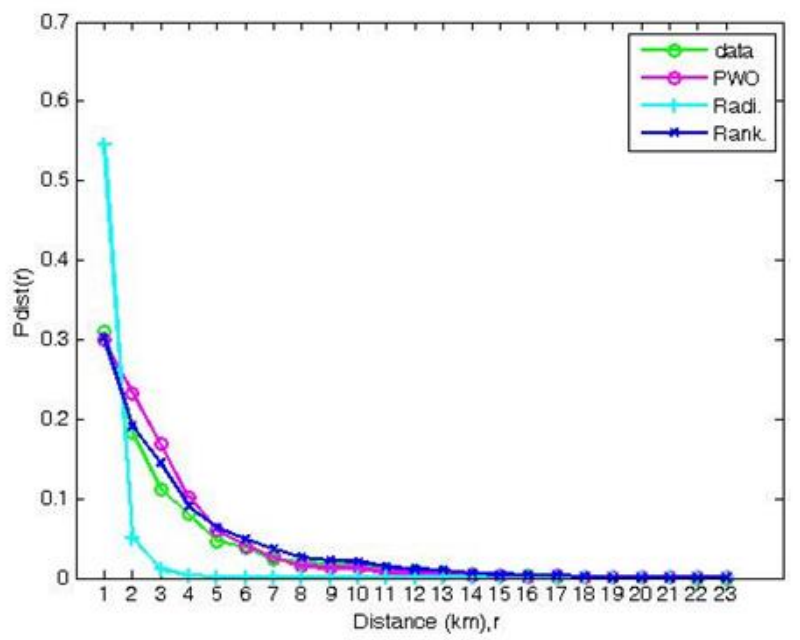

Figure 2. Travel distance distribution $P_{\text {dist }}(r)$ produced by the mentioned models in comparison with ground truth data. Here, $P_{\text {dist }}(r)$ is defined as the probability of travel between locations at distance $\mathrm{r}$.

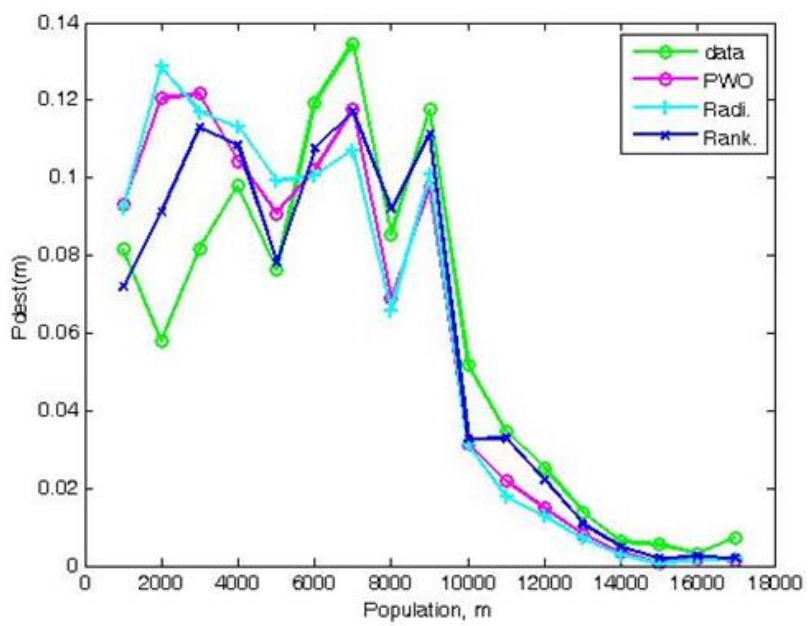

Figure 3. Comparing the destination travel constraints mentioned models with ground truth data. $P_{\text {dest }}(m)$ is the probability of travel to a location with population $m$.
Table 1: Cosine and Sørensen similarities between estimated OD matrices and ground truth data

\begin{tabular}{|c|c|c|}
\hline Model & Cosine Similarity & $\begin{array}{c}\text { Sørensen } \\
\text { Similarity }\end{array}$ \\
\hline PWO & 0.688 & 0.449 \\
\hline RANK-BASED & 0.674 & 0.49 \\
\hline RADIATION & 0.707 & 0.417 \\
\hline
\end{tabular}

\section{CONCLUSION}

This study examines the efficiency of LBSN data in the estimation of the intra-urban OD matrix in Manhattan, the one of the five boroughs of New York city. In this paper, the check-ins data from the leading LBSN provider, Foursquare, were used to analyze the intra-urban movement, and MTA origin-destination survey data served as the ground truth data to evaluate the performance of the proposed methodology. With respect to traditional and emerging travel demand data collection technologies, LBSN data has potential to investigate better spatial and temporal coverage, have real-time updating capability and much lower data collection cost. In this paper, three OD estimation models have been utilized to compare their relative performance for LBSN data. To evaluate the outputs of models, the travel distance distribution and destination travel constraints were investigated for three models based on ground truth data. The results show that the rank-based model has a better agreement with the ground truth data than those of the other models. Moreover, two indices, Cosine and Sørensen similarities, were used to measure the degree of similarity between reproduced travel matrices and empirical observations. According to the results, we found that the rank-based model has a better performance when using LBSN data than the other models. These results prove the assumption that intervening opportunity is more promising than geographical distance. In other words, in the development of future models for predicting intra-urban movement, it is essential to pay more attention to effects of the number of the closer places.

\section{REFERENCES}

Jin, P. J., Yang, F., Cebelak, M., Ran, B., \& Walton, C. M., 2013. Urban travel demand analysis for Austin TX USA using locationbased social networking data. In TRB 92nd Annual Meeting Compendium of Papers.

Jin, P., Cebelak, M., Yang, F., Zhang, J., Walton, C., \& Ran, B., 2014. Location-Based Social Networking Data: Exploration into Use of Doubly Constrained Gravity Model for OriginDestination Estimation. Transportation Research Record: Journal of the Transportation Research Board, (2430), pp. 7282.

Iqbal, M. S., Choudhury, C. F., Wang, P., \& González, M. C., 2014. Development of origin-destination matrices using mobile phone call data. Transportation Research Part C: Emerging Technologies, 40, pp. 63-74.

Calabrese, F., Di Lorenzo, G., Liu, L., \& Ratti, C., 2011. Estimating origin-destination flows using mobile phone location data. IEEE Pervasive Computing, 4(10), pp. 36-44. 
Zipf, G. K., 1946. The P1 P2/D hypothesis: On the intercity movement of persons. American sociological review, pp. 677686.

Stouffer, S. A., 1940. Intervening opportunities: a theory relating mobility and distance. American sociological review, 5(6), pp. 845-867.

Simini, F., González, M. C., Maritan, A., \& Barabási, A. L., 2012. A universal model for mobility and migration patterns. Nature, 484(7392), pp. 96-100.

Noulas, A., Scellato, S., Lambiotte, R., Pontil, M., \& Mascolo, C., 2012. A tale of many cities: universal patterns in human urban mobility. PloS one, 7(5), e37027.

Yan, X. Y., Zhao, C., Fan, Y., Di, Z., \& Wang, W. X., 2014.

Universal predictability of mobility patterns in cities. Journal of The Royal Society Interface, 11(100), 20140834. 